Exp. Brain Res. 31, 155-162 (1978)

\title{
Independent Biaxial Reorganization of the Retinotectal Projection: A Reassessment
}

\author{
J. T. Schmidt ${ }^{1}$ and S.S. Easter, Jr. \\ Biophysics Research Division and Division of Biological Sciences, University of Michigan, Ann \\ Arbor, MI 48109, USA
}

\begin{abstract}
Summary. It has been previously suggested that the retinotectal projection can reorganize independently along two orthogonal tectal axes. This possibility was reexamined by removing roughly a quarter of the retina and slightly less than a quarter of the tectum. In the tectal case, the unseated fibers arborized rostral to the ablation, but not lateral to it, and the projection shifted irrespective of tectal axes to maintain topographic order and a roughly uniform representation of retinal areas. In the retinal case, expansion into the denervated quadrant was only from the rostral, never from the medial or lateral directions. Analysis of the movements of fiber arbors shows that they respond to local competition for tectal space rather than following tectal axes.
\end{abstract}

Key words: Goldfish - Retinotectal reorganization - Competitive innervation

The early experiments of Attardi and Sperry (1963) and Jacobson and Gaze (1965) appeared to establish the immutability of the retinotectal projection in goldfish. In the first of these, portions of the retina were removed and the optic nerve crushed, and the regenerating fibers arborized only at the sites previously occupied, often bypassing other sites vacated as a result of the retinal lesion. In the second report, the medial or lateral half of one tectal lobe was removed and the nerve crushed, and the subsequent retinotectal projection originated only from the part of the retina which had previously projected to the remaining hemi-tectum. Neither case demonstrated any capacity for modifying the retinotectal map.

Later Gaze and Sharma (1970) reported that, within months after the caudal half tectum was removed, the entire retinal projection became compressed onto the remaining hemi-tectum. They concluded from this and the earlier study

1 Present address: Dept. Anatomy, Vanderbilt University Medical School, Nashville, TN 37232, USA 
(Jacobson and Gaze, 1965) that the two tectal axes differed; compression was possible rostrocaudally but not mediolaterally.

This compromise was rejected by Yoon (1971) who suggested that the failure to demonstrate mediolateral compression stemmed from the surgery. Ordinarily, the retinal fibers which terminate in the dorsal tectum enter rostrally and course, like the rays of a fan, toward their terminations (Leghissa, 1955). Yoon contended that the medial and lateral lesions, which extended to the rostral pole of the tectum, might have prevented many fibers from entering. Accordingly, he spared the rostral pole and removed less than a quarter of the tectum, in the caudomedial quadrant. Following this procedure, the retinotectal projection reorganized so that the dorsalmost portion of the visual field shifted laterally, from its normal position at the medial boundary of the tectum, to the medial edge of the lesion. Likewise, some areas of the visual field sometimes projected nearer the rostral pole than before. These shifts were interpreted as independent and simultaneous compression along the two tectal axes.

The notion of two independent orthogonal axes across both the retina and the tectum is rooted in Sperry's $(1943,1963)$ theory of neuronal specificity. Experimentally, two such retinal axes appear to be independently established at different developmental stages, both in salamanders (Székely, 1954) and frogs (Jacobson, 1968). Corresponding tectal axes have never been demonstrated (Chung and Cooke, 1975), but are assumed to direct innervation through the matching of each tectal position, determined with respect to the two tectal axes, with fibers from the corresponding retinal position, determined with respect to its two axes. Gaze and Sharma (1970) suggested that compression becomes possible if it is the relative (rather than absolute) position along the remaining tectal axis that is relevant in the matching process. If so, one might expect independent compression along the two tectal axes following quarter tectal ablation, as Yoon (1971) appears to find. However, the evidence presented was weak, since if biaxial compression did occur, two strong predictions can be made, neither of which was tested. First, the points in the visual field which previously projected to the missing tectal quadrant should, following compressive reorganization, project to two widely separate tectal areas (Fig. 1B). This seems intuitively unlikely since it requires that many of the fibers severed at the rostral boundary of the lesion must grow laterally, turn the corner around the lesion, and then run caudally, in order to terminate at a new site in the caudolateral quadrant. The second prediction concerns the magnification factor (the number of $\mu \mathrm{m}$ on the tectum per degree in the visual field). The rostrocaudal and mediolateral magnification factors (RCMF and MLMF respectively) should differ markedly in the three remaining quadrants (Fig. 1C). Specifically, the RCMF should be reduced only in the rostromedial quadrant, and the MLMF only in the caudolateral quadrant; both should remain normal elsewhere. Yoon (1971) noted decreases in the predicted regions, but there were too few points (typically 15 to 20 in the whole map) to assess it elsewhere. Double representation of some points in the visual field was suggested in one of his two maps (Fig. 7), but the issue was not treated directly.

We have reexamined the question by making similar lesions and analyzing the subsequent projections with particular attention to the predictions above. 
We used larger fish, with larger tecta, so more points could be explored, which made our maps more detailed than earlier ones. In addition we have made quarter retinal lesions and examined the subsequent reorganization in the same context.

\section{Methods}

The caudomedial quarter of the dorsal tectum was cut out with a microknife and removed by aspiration from 17 fish (12-18 $\mathrm{cm}$ body length) after anaesthetization with tricaine methanesulphonate (Crescent, Scottsdale, Arizona). The cranial incision was resealed with dental acrylic. Retinal lesions were made in five other fish using a method similar to that of Attardi and Sperry (1963). Briefly, the eye was opened at the nasal pole behind the iris and the dorsonasal or ventronasal area of both the retina and pigment epithelium was cut free with fine rat-toothed forceps. These quadrants normally project to the caudolateral and caudomedial tectal areas respectively. Remnants were removed by gentle aspiration. After the operation the eye reassumed its original shape and the ocular media cleared. The optical geometry of similarly operated eyes was investigated in a separate study by photographing frozen cross sections, and found to be very nearly normal (Schmidt et al., 1978). The retinotectal maps were determined by a method similar to that of Schwassman and Krüger (1965) which is also described in detail elsewhere (Schmidt et al., 1978). The position of the eye was continually monitored by locating the projection of the optic disc onto the hemisphere, and the maps were corrected for any shifts during the recording. Tectal penetrations were made at $300 \mu \mathrm{m}$ intervals, yielding an average of 38 points in each tectum. The maps show the correspondence between points in the visual field and on the tectum. These were corrected for the enlargement of the visual field caused by mapping in air (Schmidt et al., 1978), and used to construct the projection of the visual field meridians and $20^{\circ}$ parallels onto a standard tectum (Figs. 1A, 1E and $3 B$ ).

\section{Results}

\section{Tectal Ablations}

Eight normal fish were mapped to obtain the data in Figure 1A. The size of the tectum in each case was variable (up to $10 \%$ ), but the tectal outlines could, by differential enlargement, easily be superimposed. This allowed the data from all eight to be combined by simple averaging. Variability in the visual projection itself was small. Two of the tectally operated fish were mapped immediately after surgery, and their maps superimposed accurately onto the normal, apart from the missing region. Of the fifteen remaining tectally operated animals, six survived to be mapped from 210-464 days after the ablation; some were mapped twice. A typical map is shown in Figure 2. Nearly all of the unseated projection had moved onto the rostromedial quadrant, and no position in the visual field was doubly represented on the tectum. At early times, occasionally a displaced fiber was found in the caudolateral quadrant, but eventually maps similar to Figure 2 were obtained from all six fish.

The RCMF's and MLMF's were computed for all three remaining quadrants in the final maps and averaged. Rostromedially, the RCMF was $72 \pm 3 \%$ (mean \pm S.E.M.) of the normal value for that quadrant, a significant reduction, and evidence for compression. In the same area, the MLMF was $120 \pm 3 \%$ of the 

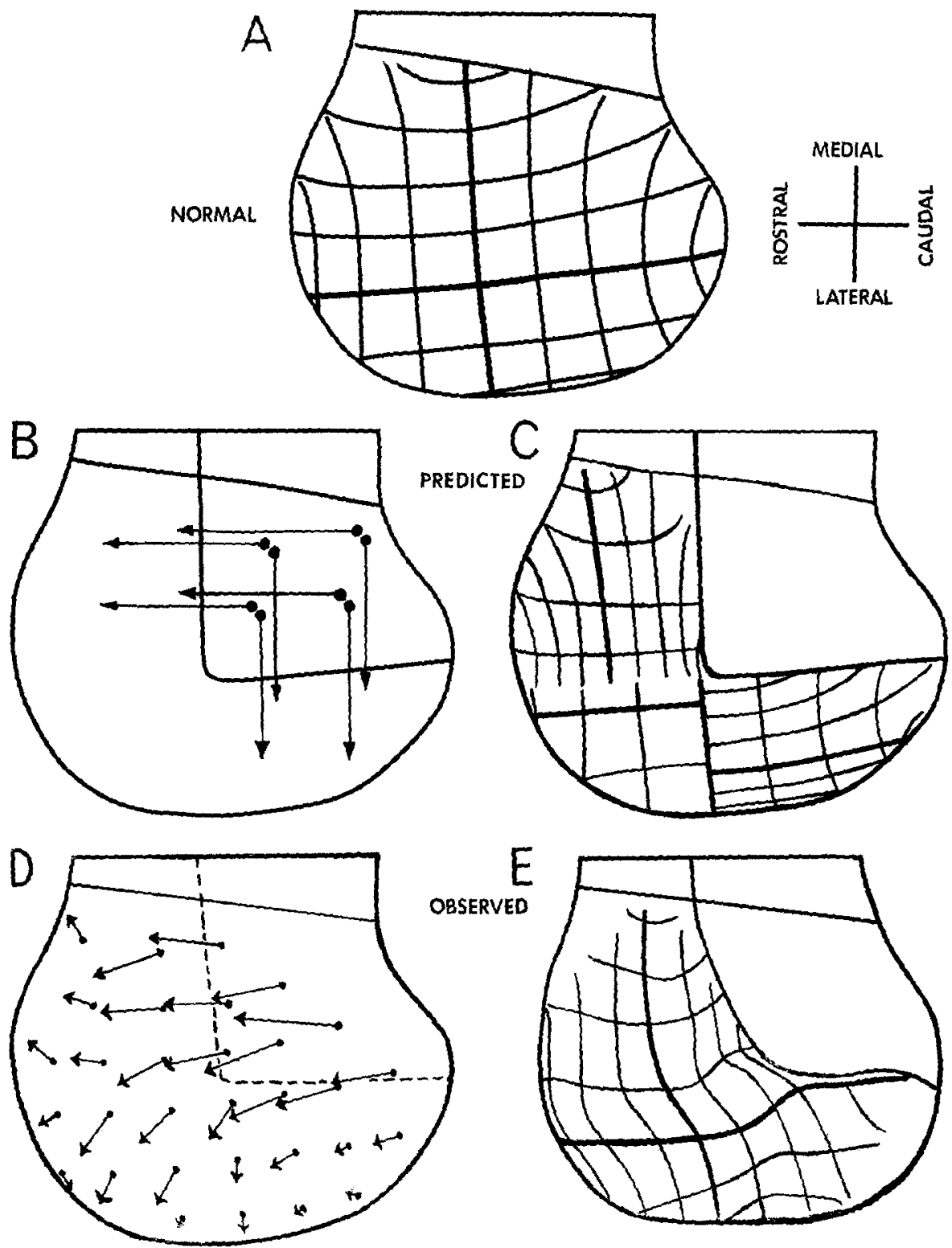

Fig. 1. Normal visual projection, and the predicted and observed projections after quarter tectal removal. A Standard tectum with meridians and $20^{\circ}$ parallels of the normal visual field projection. B Movements of fiber arbors predicted by biaxial reorganization. $\mathbf{C}$ Meridians and parallels which such a reorganization of the projection would create. D Observed movements of arbors averaged for all six experimental fish. The dashed line marks off the average extent of the tectal lesion. E Meridians and parallels of one case, that shown in Figure 2. In (A), (C) and (E), the vertical and horizontal meridians are the heavy lines 

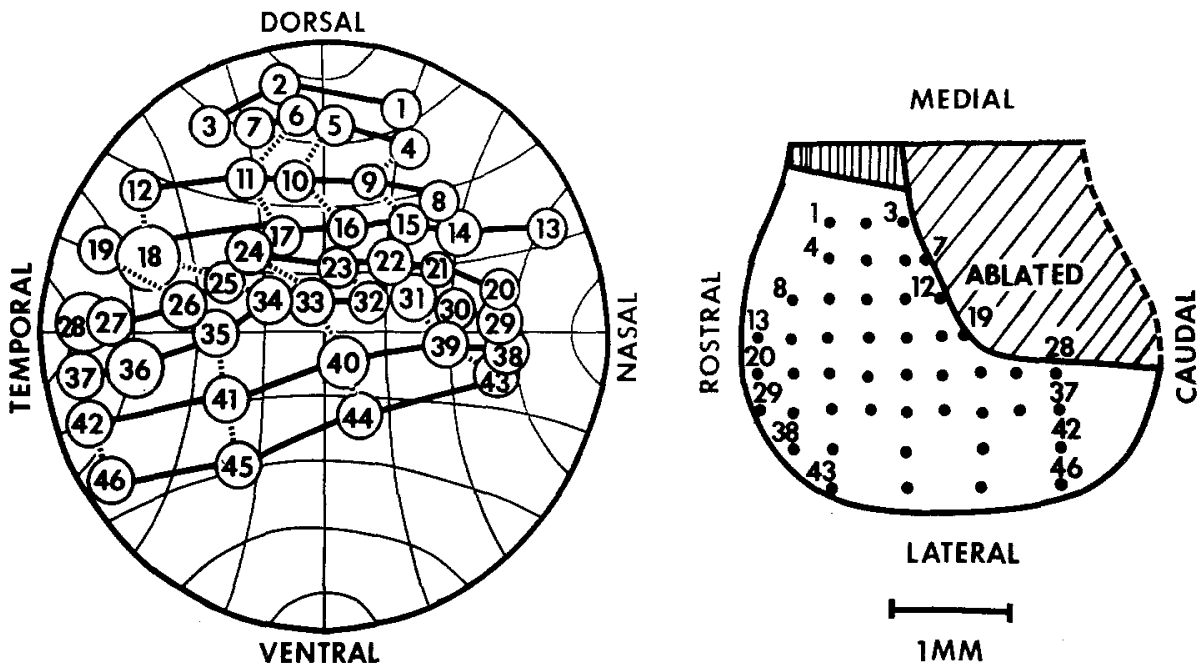

Fig. 2. The projection mapped in one fish at 293 days after the tectal lesion. The receptive fields of each rostrocaudal series of penetrations are linked by solid lines; mediolateral series are sometimes linked by dashed lines. The hemisphere is marked at $20^{\circ}$ intervals, and the circles show the sizes of the receptive fields

normal, a significant increase, and indicative of expansion. A possible reason for this expansion will become apparent later. In both the other quadrants, the MF's were insignificantly different from normal. Caudolaterally, the RCMF and MLMF were $97 \pm 8 \%$ and $96 \pm 11 \%$ of the normal value for that quadrant. Rostrolaterally, the RCMF was $93 \pm 21 \%$ and the MLMF was $96 \pm 8 \%$ of the normal value.

The nature of the reorganization became more apparent when the map of Figure 2 was transformed into the field representation shown in Figure 1E. This should be compared with the normal case in Figure $1 \mathrm{~A}$ and with the results predicted by the hypothesis in Figure 1C. Laterally, the projection of the vertical meridian lay, as in the normal, at the center of the tectum, but more medially, it curved rostrally to stay at the center of the remaining tectum there. In contrast, the projection of the horizontal meridian occupied a normal position caudally near the edge of the ablation, but shifted laterally before it reached the rostral pole. As a result, the projection of the dorsal field was both compressed rostrocaudally and expanded mediolaterally so that the tectal areas enclosed by the projections of the $20^{\circ}$ parallels were roughly constant.

\section{Retinal Ablations}

Similar, but complementary, results were also obtained in the fish with quarter retinal ablations. These lesions denervated approximately one quarter of the 
tectum which, according to the hypothesis of biaxial reorganization, should then be invaded from both axial directions simultaneously. Such an invasion would create a zone of double projection wherein each tectal point would have receptive fields from two separate retinal quadrants. In the three fish which lived to be recorded at 171-220 days, none of this was observed. Expansion into the denervated tectal quadrant was always from the rostral tectum. At no penetration in the previously denervated tectal area was there any evidence of dual projection, or indication of mediolateral expansion. The projected meridians of a typical case are shown in Figure 3B. They illustrate how the vertical parallels of the projection turned gradually at the boundary of the previously denervated quadrant to maintain a continuous topographic projection, in spite of the expansion.

\section{Discussion}

These results clearly fail to show the phenomena predicted by the hypothesis of independent biaxial compression. A more satisfactory explanation can be obtained by considering, on an individual basis, the displacements on the tectum of sites in the visual field. We assume that the microelectrode records from the terminal arbors of fibers originating near each other in the retina (Lettvin et al., 1960; George and Marks, 1974). Therefore when the same point in the visual field is represented at different tectal locations in the experimental and normal animals, that must indicate that the terminal arbor of a retinal fiber has shifted its tectal location. Such shifts can be obtained graphically from the field representations (Figs. $1 \mathrm{~A}$ and $1 \mathrm{E}$ ), and the average vector shifts for all six fish with the tectal ablations are represented in Figure 1D. In all cases, the arrow originates at the normal tectal location of one visual field position and terminates at the site where that same visual field position was mapped (on the average) in the six experimental animals.

Figure 1B shows the shifts predicted by the hypothesis. The tectum is seen to resemble a woven fabric, in which independent biaxial adjustments represent shifts along the warp and woof. Figure 1D shows the actual displacements which are obviously much less orthogonal than predicted. We suggest the following interpretation. Retinal fibers which ran toward the ablated region came from the rostral pole (Leghissa, 1955), and therefore their axons were severed at the rostral boundary of the lesion. They regenerated from this site, but finding no caudal tectum to innervate they created an artificially high density of fiber terminals near the rostral boundary of the lesion. As they arborized, they displaced the arbors already located in that region. These in turn arborized more rostrally where the arbor density was lower (that is, normal), and thus displaced others from their positions. This process of sucessive displacement of normally spaced arbors by more tightly packed ones continued in whatever directions led to a more uniform distribution of arbors. Thus the arbors were not only displaced rostrally, but also laterally, within the rostral tectum, accounting for the increased medio-lateral magnification factor in that region. However, even though competition can explain the arbor movements both out of the ablated 

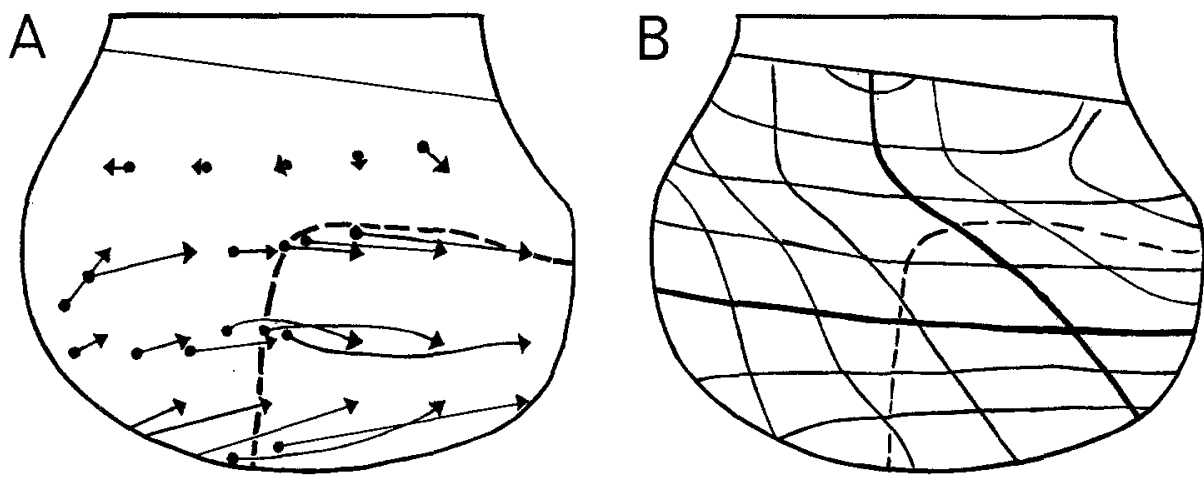

Fig. 3. Observed movements of the arbors (A) and the projected meridians and parallels (B) of one of the quarter retinal fish at 217 days postoperative. The dashed line in (B) demarcates the approximate extent of the tectal surface originally denervated by the retinal lesion

region and within the rostral area, it cannot entirely account for those in the caudolateral quadrant, where there was an unexpected and consistent movement of the arbors rostrally. At the medial edge of the ablation these movements may reflect fibers which were cut while traveling into or through the ablated area and which first rearborized rostrally, then moved laterally around the corner. However, the rostral movement of arbors far from the cut edge cannot be explained in competitive terms. These may represent synergistic movements necessary to preserve the retinotopic nature of the projection. Because of these movements, the vertical parallels (Fig. 1E) do not curve so drastically at the corner, but rather slant continuously along the diagonal.

For the quarter retinal case, the actual movements of the arborizations were also computed as before, and these are plotted in Figure 3A. They can be explained in a manner analogous to the movements which occurred after quarter tectal ablations. The retinal fibers' arbors which lay adjacent to the denervated tectum were presented with a region free of optic arbors. Accordingly, they shifted their arbors into it, leaving a relatively low density of arbors where they had been. Arbors adjacent to this area then moved into it, and so the process was repeated, ending only when the arbor density was everywhere uniform. (We have no reason to believe that this process was complete at the time of our recordings in these fish.) The observation that expansion was greater rostrocaudally than mediolaterally suggests that there is a difference between the two axes, as originally suggested by Gaze and Sharma (1970). The axes themselves can bend to the diagonal (Figs. 1E and 3B), leading us to suggest that the difference results only from the fact that the fibers normally course rostrocaudally.

In summary, we have shown that modifications of the retinotectal projection do not occur independently along two orthogonal axes. Instead, the arbors of the retinal fibers appear to move in response to more local phenomena, moving in any direction, whether parallel to either axis or diagonal to both, and thereby 
deploying across the remaining tectal surface in roughly even density while maintaining retinotopic order.

This conclusion is supported by the results from surgically created 'compound tecta' (Sharma, 1975). In that case, a normal projection was eventually established in spite of the reversal of tectal polarity within the grafted half tectum. Similar projections have sometimes been found over compound tecta in frogs as well (Jacobson and Levine, 1975). A system of orthogonal axes probably plays a role in the organization of the retina during development and the sorting out of its fibers within the optic tract and tectum, but retinal gradients do not appear to be rigidly matched with corresponding tectal axes. The latter might develop in the tectum only after retinal innervation (Chung and Cooke, 1975).

This work was supported by PHS grants EY-00168 (to S. S.E.) and GM-1355.

\section{References}

Attardi, D. G., Sperry, R. W.: Preferential selection of central pathways by regenerating optic fibers. Exp. Neurol. 7, 46-64 (1963)

Chung, S.-H., Cooke, J.E.: Polarity of structure and of ordered nerve connections in the developing amphibian brain. Nature (Lond.) 258, 126-132 (1975)

Gaze, R.M., Sharma, S.C.: Axial differences in the reinnervation of the goldfish optic tectum by regenerating optic nerve fibres. Exp. Brain Res. 10, 171-181 (1970)

George, S.A., Marks, W.: Optic nerve terminal arborizations in the frog, shape and orientation inferred from electrophysiological measurements. Exp. Neurol. 42, 467-482 (1974)

Jacobson, M.: Development of neuronal specificity in retinal ganglion cells of Xenopus. Develop. Biol. 17, 202-218 (1968)

Jacobson, M., Gaze, R.M.: Selection of appropriate tectal connections by regenerating optic nerve fibers in adult goldfish. Exp. Neurol. 13, 418-430 (1965)

Jacobson, M., Levine, R.L.: Plasticity in the adult frog brain: Filling in the visual scotoma after excision or translocation of parts of the optic tectum. Brain Res. 88, 399-410 (1975)

Leghissa, S.: La structura microscopia e la citoarchitectonica del tetto ottico dio pesci teleosti. Z. Anat. Entwickl.-Gesch. 18, 427-463 (1955)

Lettvin, J. Y., Maturana, H. R., McCulloch, W. S., Pitts, W. H.: Anatomy and Physiology of Vision in the Frog (Rana pipiens). J. Physiol. (Lond.) 43, 129-175 (1960)

Schmidt, J.T., Cicerone, C. M., Easter, S. S.: Expansion of the half retinal projection to the tectum of goldfish: An electrophysiological and anatomical study. J. comp. Neurol. (In press) (1978)

Schwassman, H.O., Krïger, L.: Organization of the visual projection upon the optic tectum of some freshwater fish. J. comp. Neurol. 124, 113-126 (1965)

Sharma, S.C.: Visual projection in surgically created compound tectum in adult goldfish. Brain Res. 93, 497-501 (1975)

Sperry, R. W.: Visuomotor coordination in the newt (Triturus viridescens) after regeneration of the optic nerves. J. comp. Neurol. 79, 33-55 (1943)

Sperry, R.W.: Chemoaffinity in the orderly growth of nerve fiber patterns and connections. P.N.A.S. (USA) 50, 703-709 (1963)

Székely, G.: Zur Ausbildung der lokalen funktionellen Spezifität der Retina. Acta biol. Acad. Sci. hung. 5, 156-167 (1954)

Yoon, M. G.: Reorganization of the retinotectal projection following surgical operations on the optic tectum in goldfish. Exp. Neurol. 33, 395-411 (1971) 